\title{
Self-assembly of biopolymer films for UV protection of wood
}

\author{
Kristina Ivana Fabijanic ${ }^{1, a)}$ (1), Aída Ninfa Salinas López ${ }^{1}$, Long Pan ${ }^{1}$, Chi-Yuan Cheng ${ }^{1}$, \\ Yu Wang ${ }^{1}$, Zhigang $\mathrm{Hao}^{1}$, Andrei Potanin ${ }^{1}$, Ningwei Li $^{1}$, Isaac Rangel ${ }^{1}$ \\ ${ }^{1}$ Colgate-Palmolive Technology Center, Piscataway, NJ, USA \\ a) Address all correspondence to this author. e-mail: Kristina_Fabijanic@colpal.com
}

Received: 29 April 2021; accepted: 10 August 2021; published online: 12 October 2021

\begin{abstract}
There is an increasing need for materials with tunable physical and chemical properties that are relatively non-toxic and efficacious for their intended application. Many wood stains and finishes emit toxic chemicals which may have serious implications to one's health. A novel alternative material is realized between xanthan gum and Neodol, a non-ionic surfactant. The resulting three-dimensional film is evaluated as a free-radical scavenger for the protection of wood at different ratios. Atomic force microscopy visualizes the topography and quantifies the local nanomechanics, while rheological measurements showcase a shift from viscoelastic material to gel. Electron plasmon resonance confirms the free-radical reducing ability (3.5 times), while liquid chromatography mass spectroscopy quantifies the UV degradation of sinapyl alcohol. This material has potential, not only in coating industries as a safer option, but also in those industries requiring flexibility and tenability, namely for biosensors and antiinflammatory therapeutics.
\end{abstract}

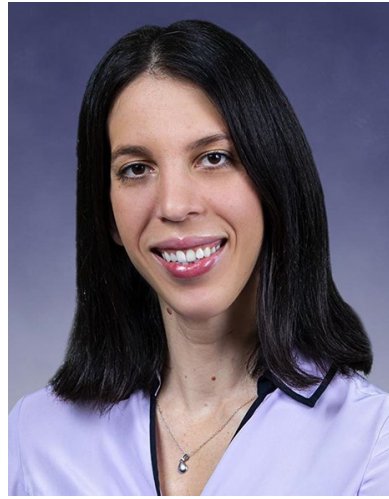

Kristina Ivana Fabijanic
Kristina Ivana Fabijanic has been a part of Colgate-Palmolive Co. since July 2018. She received her B.S. in Chemistry from Fordham University in 2007 and completed her Ph.D. in Nanotechnology and Materials Science from CUNY Graduate Center/CUNY Hunter College in 2014. After completion of graduate school, she worked as post-doctoral researcher at CUNY Hunter-Weill Cornell until 2017. She had also worked at CUNY Lehman College. Fabijanic's past work focused on roomtemperature synthesis of semiconductors with enzymes, SAMs and micro-contact printing. She also investigated targeted drug-delivery for cancer therapeutics and mechanical characterization of cells and extracellular vesicles for correlative metastatic studies with Atomic Force Microscopy. Her current research interests include the development of screening techniques to innovate, predict and dive-deep to understand how the local and bulk mechanics of different materials are altered upon their interaction with various ingredients found in consumer products.

\section{Introduction}

Recently, there has been an increase in focus around the selfassembly of hydrophilic biopolymer networks owing to their more environmentally friendly nature and naturally derived sourcing toward the production of functional materials like hydrogel polymeric films and coatings $[1,2]$. Hydrogels are an interesting class of materials due to their flexibility and mechanical strength owing to their architectural properties [3]. The spread of potential applications using polymeric films span from tissue engineering and drug delivery across the blood-brain barrier, wound healing dressing, use in the optical biosensor industries, and even include applications centered around biodegradable packaging, anti-fogging, or anti$\mathrm{UV}$, which is where the focus of this study is directed [4-12]. 
Polymeric-based films are being investigated more intently recently because they combine advantages from two or more materials as their name implies [13]. Usually, their controllable pore sizes and thicknesses can be used in a multitude of applications. The three-dimensional self-assembly of films with tunable properties from the macroscale to the nanoscale are being studied more extensively due to their immense potential cross category. The hindrance in making the switch to use a more environmentally sustainable material source, however, is the inability to control crucial parameters depending on which application one is focused on. For drug delivery, it is the inability to control pore size, whereas for anti-UV it may be a lack of mechanical strength. Some of the current methods for producing hydrogel polymeric films include, but are not limited to freeze-thaw, electro-spinning, porogen leaching, or $\mathrm{CO}_{2}$ gas-foaming [14-16]. As a whole, these methods allow for the formation of interesting structures, but, some outlying issues include poor control over pore size, lack of mechanical strength, long-processing times, or unrealistic reactor qualifications [17]. Electro-spinning has also been used to produce sub-micron porous assemblies composed of PVA and polyacrylic acid [18]. While each of these processes used individually or in combination can control different aspects of the resulting structure, several setbacks continue to persist.

Xanthan gum is an anionic heteropolysaccharide secreted by the bacteria Xanthomonas campestris and produced by the fermentation process [19]. Its primary structure consists of repeating pentasaccharide units formed by two glucose units, two mannose units, and one glucuronic acid unit and is generally used for thickening aqueous solutions, stabilizing emulsions and dispersing properties [20]. Antioxidant properties of xanthan gum have been reported in the past in substrates such as ram sperm, different foods, corneal epithelial cells, and lipid model systems [21-24]. However, this is the first time that UV protection in wood has been mentioned as a property of this material when hydrogen bonded with a C9-11 ethoxylated alcohol. This natural polymer material requires no directed self-assembly and simply forms at the precise ratio between xanthan gum and Neodol through physical crosslinking. It has been shown that xanthan gum can hydrogen bond with non-ionic surfactants such as Tween 80 through hydrogen bonding in the bulk [25]. The use of chemicals such as glutaraldehyde or epichlorohydrin are not required which removes the toxic component and adds to its environmental benefits [26]. By using hydrogen bonding between two simple and readily available ingredients, the self-assembled 3-dimensional hydrophilic gel network can be fine-tuned depending on desired material properties. Two substrates, mica and cellulose filter paper, resulted in a shift in optimum ratios which allowed for the development of a rapid prediction method to more quickly predict optimum ratios on cellulose-mimicking substrates. This work shows how this bioavailable and biodegradable material with controllable parameters can be used in varying industries depending on the outcome desired-we will focus on showing the free-radical scavenging effect for coating purposes based on the increased crosslinking effect of UV irradiation.

\section{Results and discussion}

\section{Nano-scale characterization of films via atomic force microscopy}

The self-assembly of biopolymer films is achieved through the mixing of the xanthan gum (XG) and Neodol (Neo), in deionized water. As the ratio of Neo relative to XG is increased, the formation of pores is observed as is an increase in both size and strand thickness. The ratios of XG-Neo (w/v) examined are $4.5-1,1-1,1-4.5,1-6.5,1-11.1$, and 1-22.2. Pore formation is first observed at the 4.5 parts XG and 1 part Neo ratio. First, the thickness of the film is evaluated. As seen in Fig. 1a, the biopolymer film on the mica substrate has a total height of $1.98 \mu \mathrm{m}$. (a)

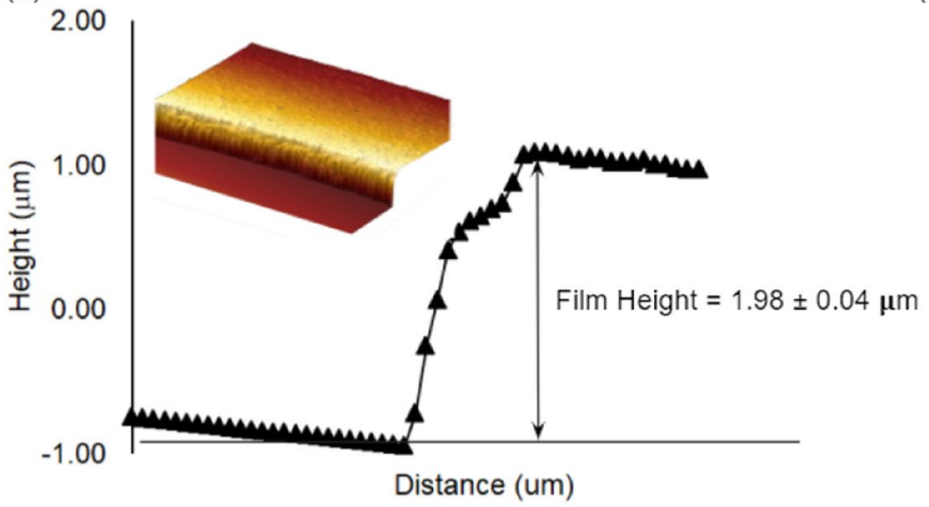

(b)

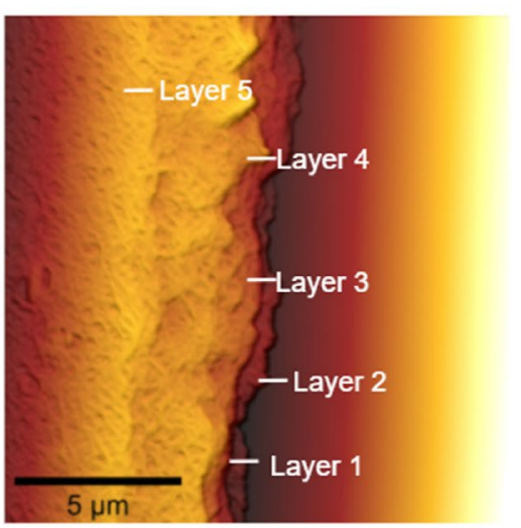

Figure 1: Layer-by-layer film deposition at interface for 1 part Neo and 6.5 parts XG. (a) Inset showing $100 \mu \mathrm{m} \times 100 \mu \mathrm{m}$ atomic force microscopy (AFM) 3D height image of the film interface on mica and an average height of $1.98 \mu \mathrm{m}$. (b) $15 \mu \mathrm{m} \times 15 \mu \mathrm{m}$ atomic force microscopy height image of the film interface showing different layers achieved through controlled sample volume deposition and drying time. 
(a)

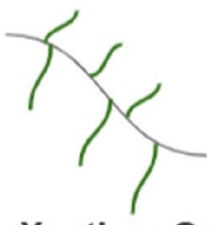

Xanthan Gum (e)

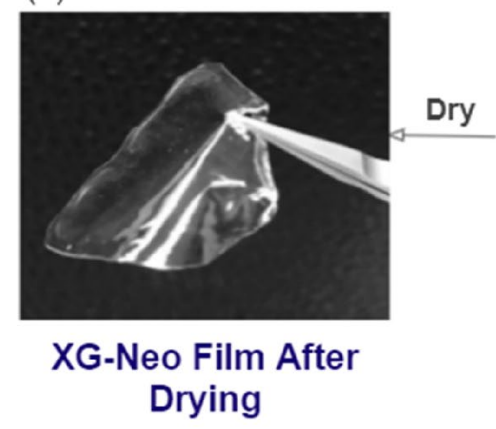

(c)

$\left.\right|_{\nabla}$ I (b)

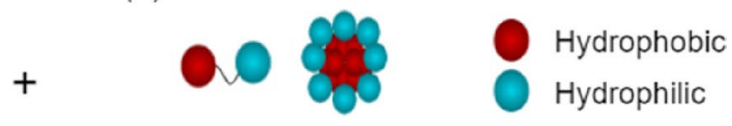

\section{C9-C11 Ethoxylated Alcohol}

(b) 808 Hydrophobic

(1)

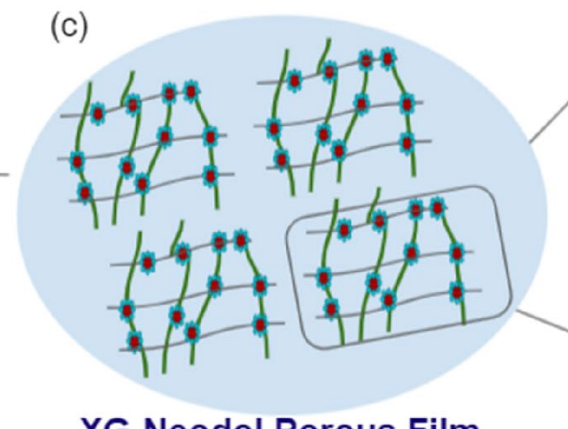

XG-Neodol Porous Film (d)

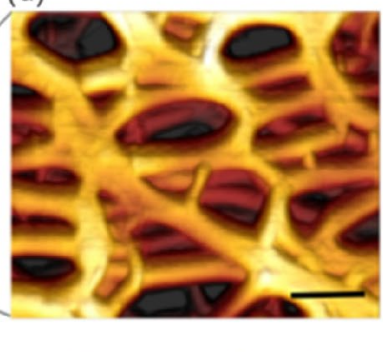

Topography of Biopolymer Film

Figure 2: Fabrication of porous biopolymer films from the polysaccharide, xanthan gum and the ethoxylated alcohol, Neodol. (a) Schematic representation defining the structure of the polysaccharide, xanthan gum and (b) Neodol which may exist in both spherical and chain-like micelles depending on concentration. (c) Illustration of the self-assembled porous network that forms in-situ via hydrogen bonding. (d) AFM topography of $1.5 \mu \mathrm{m} \times 1.5 \mu \mathrm{m}$ area showing porous network; scale bar $300 \mathrm{~nm}$. (e) Macroscopic view of the film after drying at room temperature.

The layer-by-layer deposition is done under controlled volume and drying time with the result shown in Fig. 1b. It is determined that each layer is within a close range to the former and latter layers, on average, $363.34 \mathrm{~nm}$. Knowing the starting height of the films is necessary for performing later tests on the elastic modulus of the material. If the thickness of the soft film is small, care must be taken with regard to two points: (1) If the film is extremely thin such that the tip may interact with both film material and substrate, special calibrations may be needed; (2) If the film is soft, the tip utilized should have a low spring constant and small forces need to be applied. By using low forces (nN range), a tip with a relatively low spring constant and a film with average thickness of $1.98 \mu \mathrm{m}$, the material is not undergoing irreversible deformations as is the case with nanoindentation and the use of a diamond tip [27, 28].

The ability to produce biopolymer films with different pore sizes and controllable film thicknesses seems to tremendously improve on an aforementioned setback-control. Figure 2 shows the general process for the self-assembly of XG and Neo. Xanthan Gum obtains its overall negative charge from glucuronic acid and pyruvic acid [29]. It also contains acetyl groups which are available to hydrogen bond and entangle intramolecular as well as in an intermolecular fashion [30]. Neo is an ethoxylated alcohol composed of a C9-C11 chain and is non-ionic. The surfactant is used at concentrations between 1 and $2 \%$ for experiments, which is well within the critical micelle concentration (CMC) (Supplemental Material). At this concentration, Neo exists as a micelle structure, which may allow for further stabilization and interaction of xanthan chains through hydrogen bonding.

As Neo concentration increases, the micelles in solution act as spacers within the xanthan gum chain. The variation in the number of micelle structures allows for control over pore size as seen in Fig. 3. As more Neo is added to the system, pore size increases (Fig. $3 \mathrm{a}-\mathrm{d}$ ). At the saturation point, pore structure begins to distort (Fig. 3e, f). Figure $3 g$ shows how the pore size seems to incrementally increase and is more sensitive to the amount of Neo in the system, presumably since it is acting as the linker between xanthan chains. While the pore size does not change dramatically after the 1-6.5 ratio, the deviation of sizes increase suggesting an increase in heterogeneity, potentially from local changes in material mechanics.

As seen in Fig. 4, the qualitative local Young's modulus is stiffest for the 1-6.5 ratio, homogeneously decreases in the 1-11.1 ratio, and heterogeneously changes in the case of 1-22.2. In the case of adhesive forces, the major change is from Fig. $4 \mathrm{~d}$, e, while little change is seen when comparing Fig. $4 \mathrm{e}$ and f. Thus, the major driving force in material mechanics at the 1-6.5 ratio seems to stem from the Young's modulus of the material, while the driving force behind mechanics in the 1-11.1 and 1-22.2 ratios are the adhesive forces. The tunability of this material allows for flexibility depending on the particular application. In one instance, a more elastic film may be beneficial, whereas for others, a more adhesive film is beneficial [31]. 
(a)

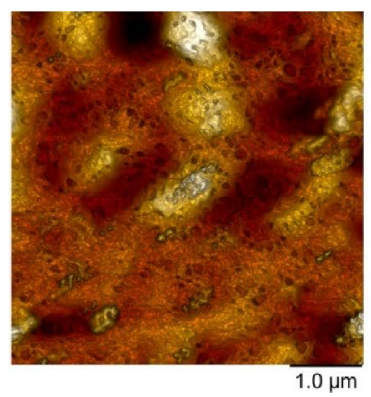

(d)

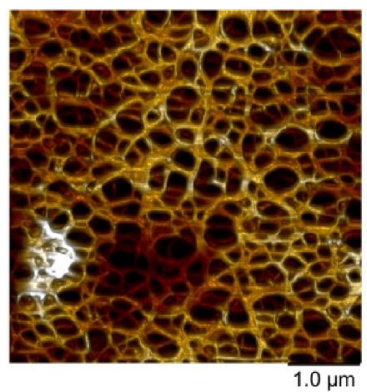

(b)

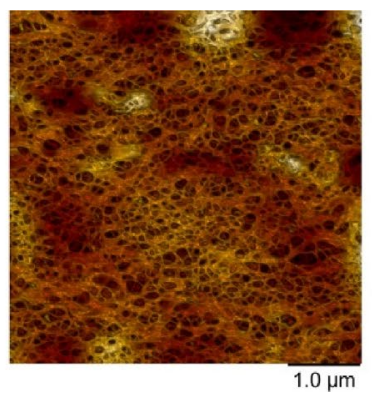

(e)

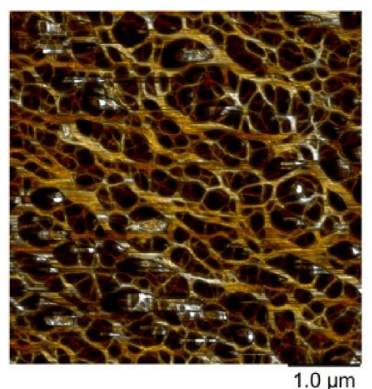

(c)

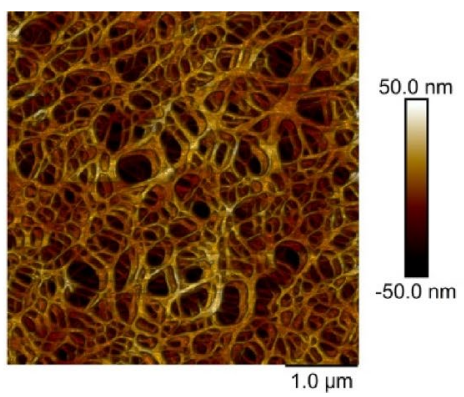

(f)

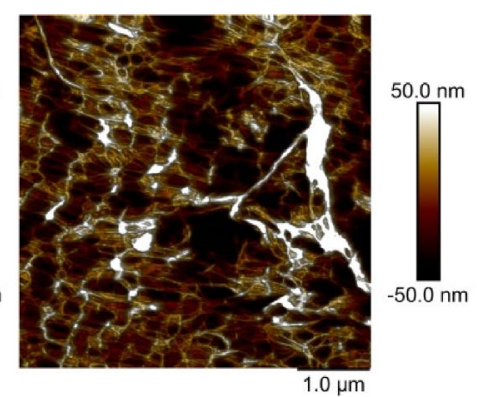

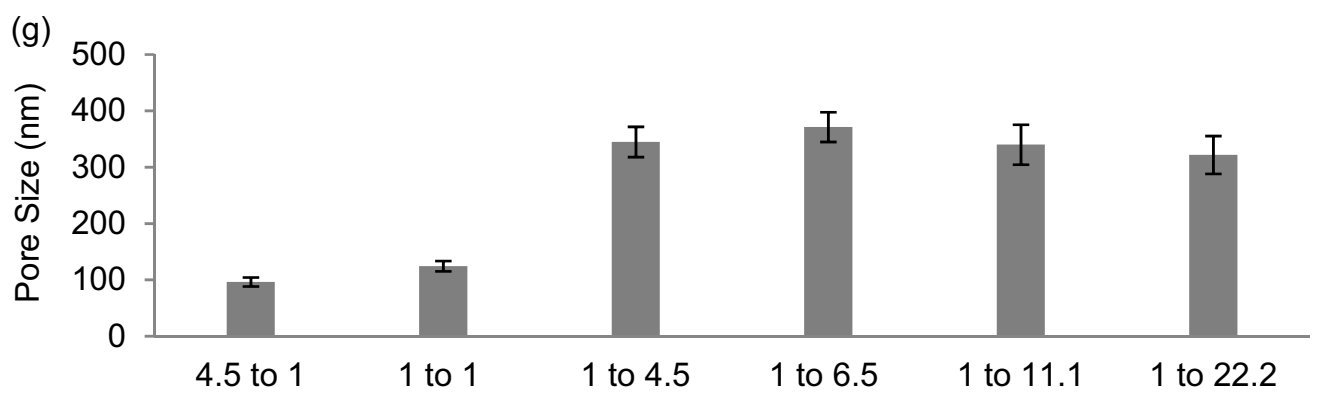

Figure 3: Varying pore size based on xanthan gum and Neodol ratios, $5 \mu \mathrm{m} \times 5 \mu \mathrm{m}$ AFM topography. (a) $4.5-1$ Xanthan gum to Neodol. (b) $1-1$ XG:Neo. (c) 1-4.5 XG:Neo. (d) 1-6.5 XG:Neo. (e) 1-11.1 XG to Neo. (f) 1-22.2 XG:Neo. (g) AFM quantitative analysis of $15 \mu \mathrm{m} \times 15 \mu \mathrm{m}$ areas showing pore size of varying ratios (error bars representing $95 \% \mathrm{Cl}$ of the mean).

While the local properties are necessary to fundamentally understand the nanostructure of the material, it is also imperative to examine the material in bulk. In Fig. 5a, left to right, the bulk liquid-film state is observed for 1-6.5, 1-11.1, and 1:22.2 (XG:Neo), respectively. As the surfactant versus xanthan gum ratio increases, the transparency of the material decreases which may be correlated to the change in pore size and mechanics as seen from AFM. Figure $5 b$ and $c$ shows the solid-film state of the liquid-film material in Fig. 5a, indicating that this material can also be potentially used in the applications involving wound dressings.

\section{Bulk mechanics via rheology}

To quantify the bulk mechanical properties of the material observed in Fig. 5, viscoelastic moduli were measured by means of frequency and temperature sweeps as described in the
"Materials and methods" section. The results of these tests are shown in Fig. 6 for the three gels at various ratios of XG to Neo. As one can see from Fig. 6a, at low-Neo level (1:6.5) the "gel" is not quite the true gel in the rheological sense, but rather a viscoelastic fluid. Only at higher Neo levels the system becomes the true rheological gel with $G^{\prime}>G^{\prime \prime}$. This indeed agrees with the visual observations depicted in Fig. 5.

Responses of these three systems to heat are shown in Fig. 6b and c. In Fig. 6b, the two low-Neo samples, 1-6.5 and 1-11.1, effectively melted (softened upon heating) at 40 and $60{ }^{\circ} \mathrm{C}$, respectively, which is indicated by $G^{\prime}$ dropping below $G^{\prime \prime}$. On the other hand, the high-Neo sample (1:22.2) did not melt at all in this temperature range. Moreover, Fig. $6 \mathrm{c}$ shows that this melting of the low-Neo samples is actually irreversible, at least within the time span of this test (20 min for the whole cycle). This may explain superior protection provided by coatings at high-Neo compositions. 
(a)

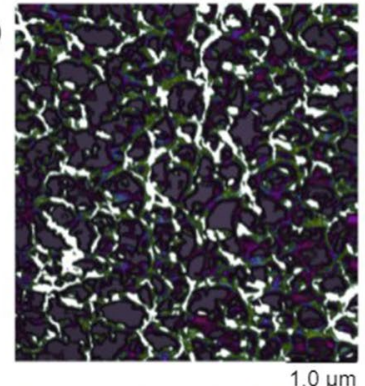

(d)

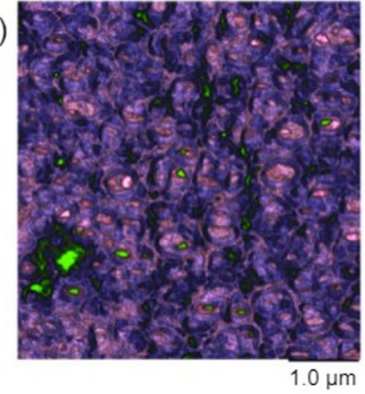

(b)

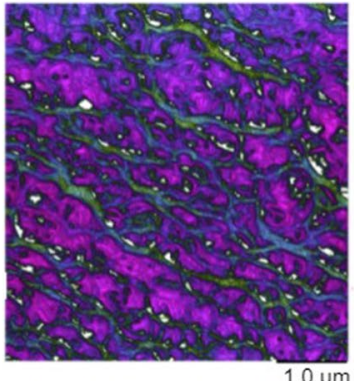

(e)

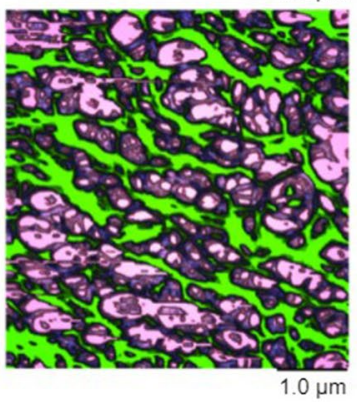

(c)

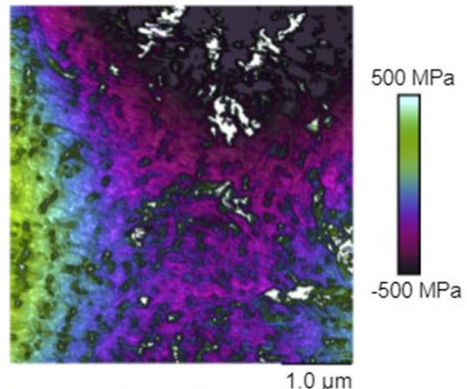

(f)

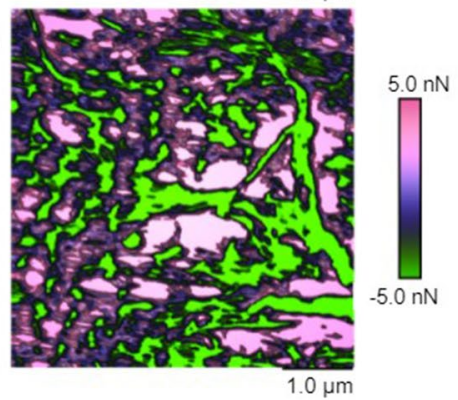

Figure 4: AFM PeakForce quantitative mechanical mapping $5 \mu \mathrm{m} \times 5 \mu \mathrm{m}$ areas. (a, d) Young's modulus and adhesive forces for $1-6.5 \mathrm{XG}$ to Neo. (b, e) Young's modulus and adhesive forces for 1-11.1 XG to Neo. $(c, f)$ Young's modulus and adhesive forces for 1-22.2 XG to Neo.

1 to 6.5

(a)

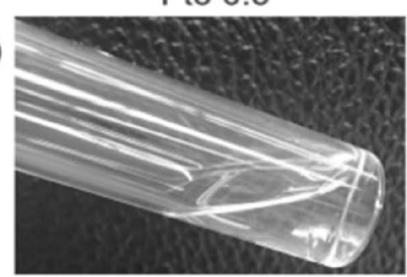

(b)

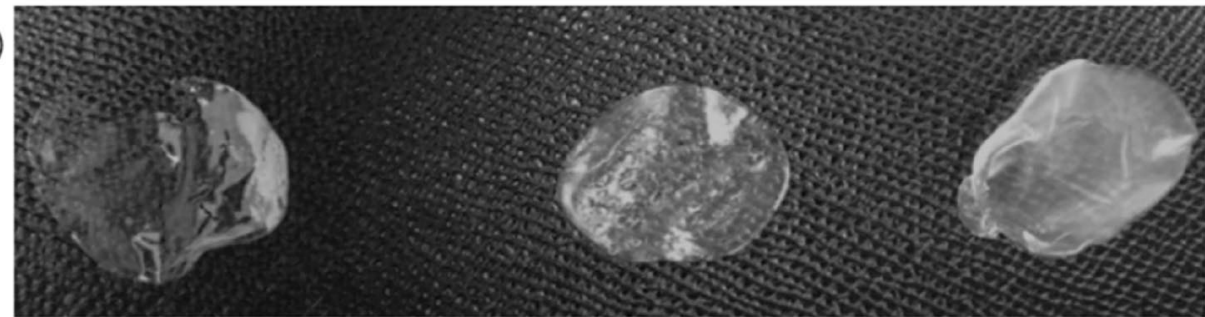

(c)

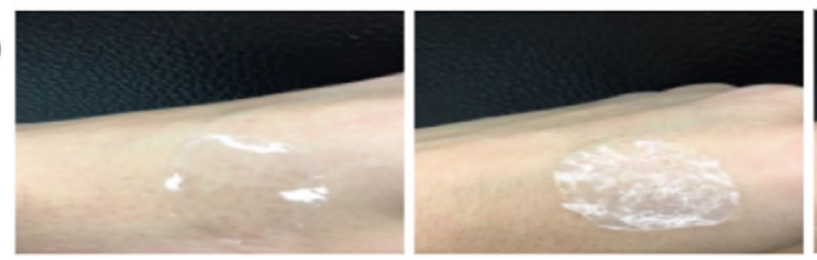

1 to 22.2
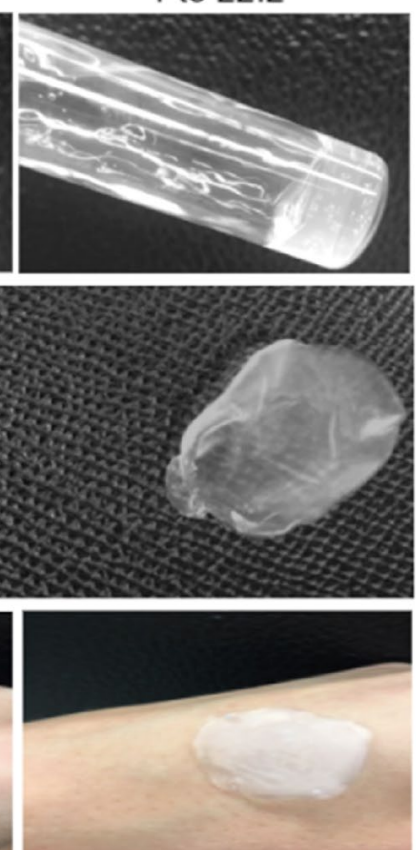

Figure 5: Macroscopic view of the viscoelastic solutions and gels before and after drying at room temperature. (a) From left to right: 1-6.5 XG to Neo solution as viscoelastic solution; 1-11.1 XG to Neo gel and 1-22.2 XG to Neo gel. (b) Films produced after taking $500 \mu \mathrm{L}$ of each solution and allowing to dry yields films of varying thicknesses, elasticities and transparencies. (c) Dry films placed on skin for an alternate view.

\section{UV protection of wood with novel films}

To evaluate the potential use of this material in the coating industry, the effectiveness of this product on wood is studied. Wood is a major part of peoples' homes, with the majority of homebuyers (54\%) willing to pay more for hardwood floors, which can last a lifetime compared to that of a carpet which has a lifetime of 10-15 years [32]. Wood is an excellent light absorber, with its cell wall being mainly composed of cellulose, 
(a) 100

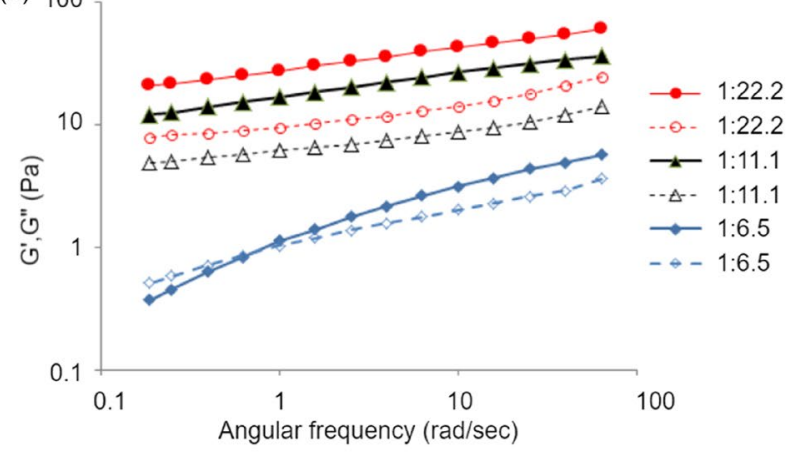

(b) 100

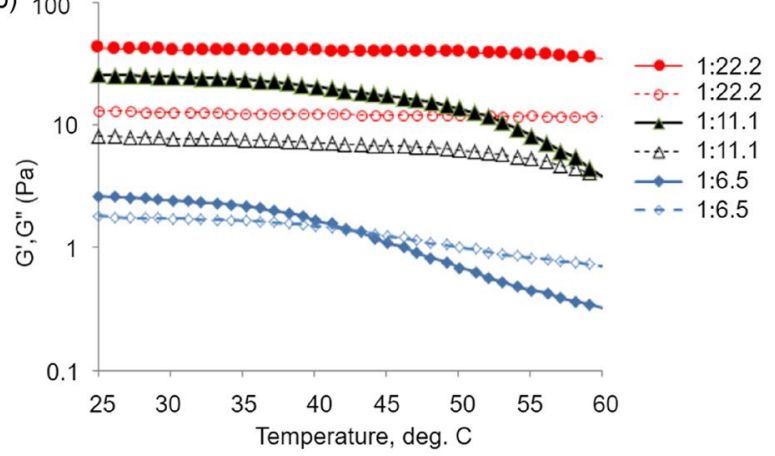

(c) 100

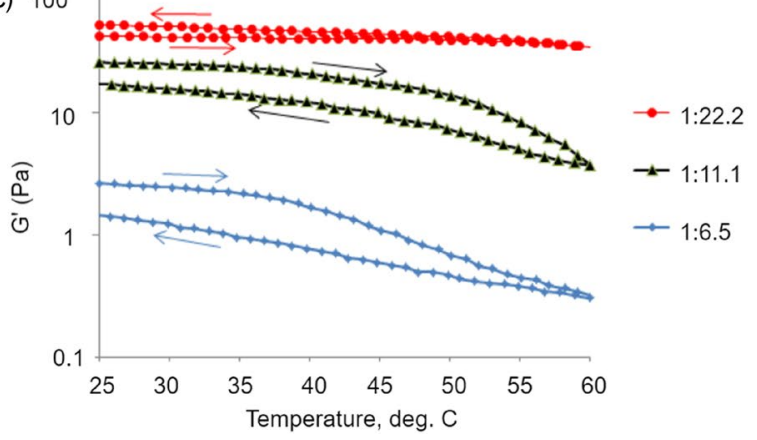

Figure 6: Viscoelastic moduli, storage (or elastic, $G^{\prime}$ - filled symbols), and loss (or viscous, $G^{\prime \prime}$ —empty symbols), are plotted against frequency (a) and temperature (b, c). Composition of the gel is shown in terms of Xanthan to Neodol ratios. Frequency sweeps were performed at $25^{\circ} \mathrm{C}$, while temperature sweeps at frequency $1 \mathrm{rad} / \mathrm{s}$. On (b) both moduli are plotted with effective melting indicated by the point at which $G^{\prime}$ drops below $G^{\prime \prime}$, which happens at $40^{\circ} \mathrm{C}$ for $1: 6.5$ system and at $60^{\circ} \mathrm{C}$ for 1:11.1. No melting at all was observed at a higher Neodol level at 1:22.2. On (c), not to overcrowd the plot, only storage modulus, $G^{\prime}$, is plotted, but both heating and cooling sweeps displayed with temperature change direction indicated by arrows so as to demonstrate irreversible (at least within the time of the experiment) melting of the low Neodol systems and no melting at high Neodol level.

hemicellulose, and lignin. When wood is exposed to sunlight, UV rays interact with lignin inducing photo-degradation. These changes are a surface phenomenon, occurring to a depth of $0.5-2.5 \mathrm{~mm}$. The absorbed energy causes dissociation of bonds of wood molecules, resulting in free radicals that cause depolymerization and formation of chromophoric groups, leading to discoloration and degradation. This discoloration and degradation will cause people to spend more money to re-stain or repair their UV-damaged wood [33].

Therefore, there exists a need for a lignin-mimicking material that can help protect fading and/or degradation in wood. Flatt and Schober agree that lignin constitutes $\sim 20-30 \%$ of the cell wall [34]. This molecule is mainly responsible for color change in the weathering cycle, as reported by Feist [33, 35]. We focused our investigation on understanding the effect involving this molecule which is formed in nature through the radical polymerization of $p$-coumaryl alcohol, coniferyl alcohol, and sinapyl alcohol, containing the $p$-hydroxyphenyl $(\mathrm{H})$, guaiacyl (G), and syringyl (S) units, respectively. Depending on the biomass source, lignin varies in monomer composition [36]. At the onset of lignification, there occurs a simultaneous copolymerization between coniferyl alcohol and tiny amounts of $p$-coumaryl alcohol. This copolymerization occurs in the primary wall to form a mixture of lignin composed mostly of $\mathrm{G}$ and $\mathrm{H}$ units. Afterwards, there is a second copolymerization reaction that occurs, this time during secondary wall development and, this time, involving $\mathrm{G}$ and $\mathrm{S}$ units $[37,38]$. Interestingly and most notably, the difference between hardwoods and softwoods is a difference between ratios that exists between $\mathrm{G}, \mathrm{H}$, and $\mathrm{S}$ components as previously shown by Santos et al. [39], specifically in the case of pine wood, which has little S unit (sinapyl) and mostly G units (coniferyl alcohol). Therefore, it will also be interesting to vary the amount of these monolignols to mimic different type of wood.

To gauge whether this material has UV-protection capabilities, it is necessary to evaluate its ability to prevent the formation of free radicals which have been shown to contribute to color change in wood. Electron plasmon resonance (EPR) measurements are able to detect free radicals produced from wood samples upon UV irradiation. The amount 
(a)

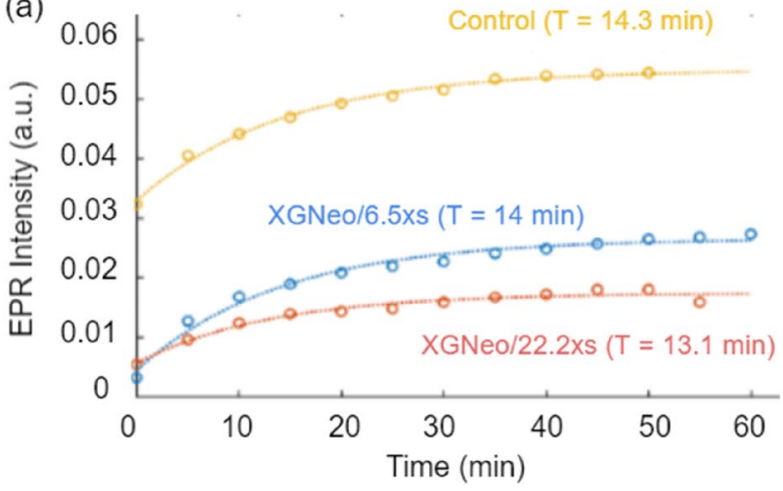

(b)

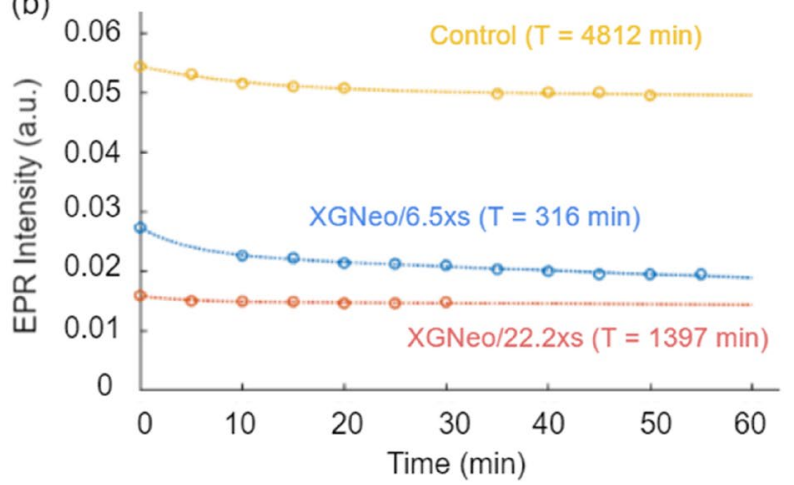

Figure 7: Electron paramagnetic resonance free-radical generation and decay. (a) UV illumination at room temperature: showing the rise of free-radical generation in untreated and treated wood with 1-6.5 and 1-22.2 times XG to Neo. (b) Post-UV dark adaptation at room temperature showing the decay of EPR signal right after turning off UV lamp.

of free radicals generated is proportional to the EPR signal intensity, as shown in Fig. 7a and b XG and Neo between the ratios of 6.5 and 22.2 , correlate to 2.5 and 3.5 reductions in the number of free radicals produced when applied to wood as compared to the control which is just untreated wood. As shown in Fig. 7b, the decay of EPR signal intensity does not really change after $1 \mathrm{~h}$, indicating that free radicals in the wood generated by UV irradiation are stable with a long lifetime. From the $\mathrm{g}$ factor of the EPR signal, we know that the EPR signals do not contribute from paramagnetic metals and is more likely coming from the carbon-centered radical from the wood structure, with a relatively longer lifetime. However, the identification of the type of free radicals is beyond the goal of this research.

To monitor film morphology post UV exposure, 1 part XG and 22.2 parts Neo are evaluated with filter paper as the substrate and imaged every $10 \mathrm{~min}$ for a total time of 20-min exposure time, as shown in the $5 \mu \mathrm{m} \times 5 \mu \mathrm{m}$ AFM images in Fig. $8 \mathrm{a}-\mathrm{c}$. $20 \mathrm{~min}$ is chosen as the final time point because, according to EPR data, that is the time-point at which the amount of free-radical generation remains constant. As suspected, the network disintegration is observed. To correlate these findings with real-world potential, a block of pine wood is treated with various ratios (Fig. 8d) and exposed to UV. After 35-h UV exposure, all ratios seem to offer some form of protection (right), with more coming from those compositions with higher-Neo content versus untreated wood (left). This finding implies that the presence of network structure correlates with function-namely protection efficiency.

\section{Liquid chromatography-mass spectrometry degradation product identification}

To successfully identify the degradation products and to further evaluate the effect of the XG:Neo effect on lignin, mass spectroscopy analysis was performed. Because lignin is a complex polymer, it was thought to try a monolignol to see if it might provide some insight into what is being formed in-situ.

Initially, the monolignol studied was sinapyl alcohol. For a more realistic mimicking system, it would be better to look at coniferyl alcohol since that is the monolignol that is present most in pine wood, but that is not reported in this work. From the total ion chromatography, the relative intensity of all the charged species in the sinapyl alcohol monomer and with added product is shown in Fig. 9. Without UV treatment, the sample with the product treatment (1:6.5 ratio) contains a higher amount of total ions compared to the one without any product treatment. However, after UV treatment for both of them, there is a significant difference in the amount of degradant/oxidized compound which is a dimer of sinapyl alcohol, this confirms that the product can protect sinapyl alcohol monomers from UV radicals.

\section{Conclusions}

We have identified a new and interesting material made through the self-assembly via an anionic polysaccharide, xanthan gum and a non-ionic surfactant, Neodol at room temperature. It has been shown that the fundamental properties of the resulting film such as pore size, modulus, and adhesion can all be controlled by fine tuning the ratio. The UV protection of wood has been evaluated as a potential application for this environmentally benign material. Depending on the desired application, smaller pore size and "softer" material may be desired. As was shown through AFM and rheology, a higher amount of Neo is necessary and seems to be less vulnerable to heat which aligns with why it produces less free radicals and has an advantageous protection of wood. 
(a)

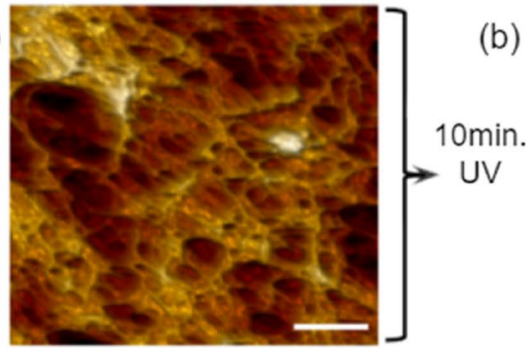

(d)

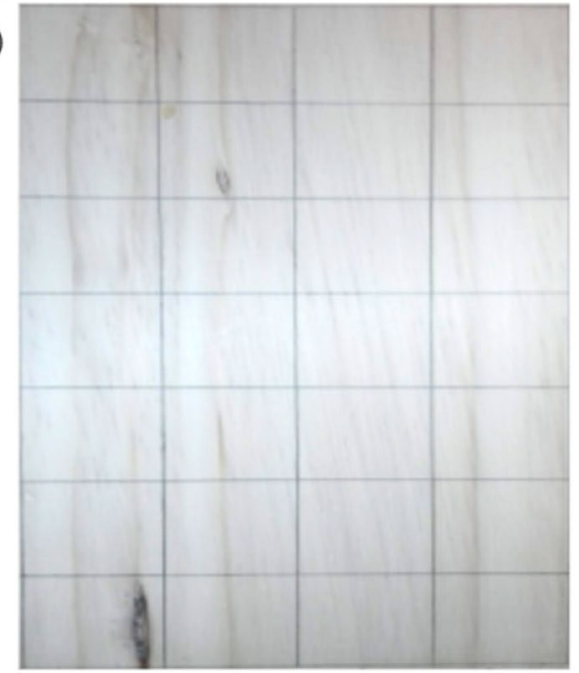

(b)

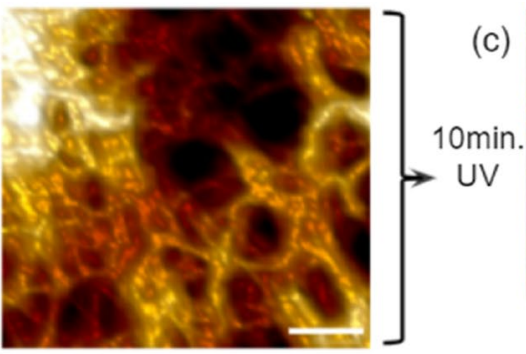

c)
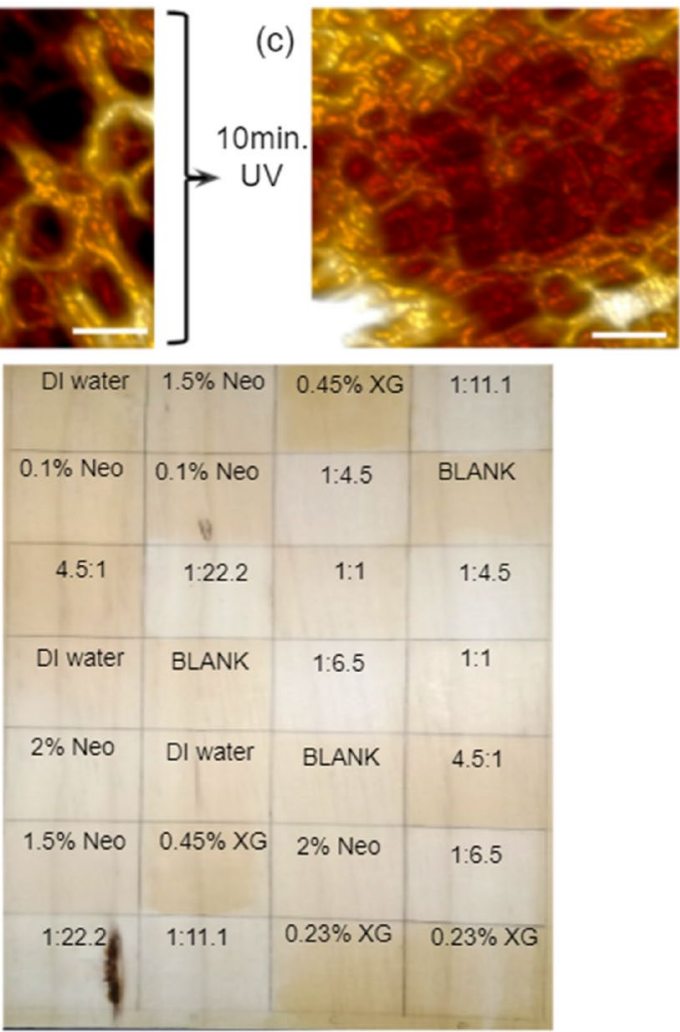

Figure 8: (a) 1:22.2 XG:Neo coating on filter paper substrate before UV exposure showing network structure integrity. (b) 1:22.2 XG:Neo on Filter Paper after 10-min UV exposure showing some loss in network structure. (c) Decreased network structure integrity after 20-min irradiation; all scale bars are $=1 \mu \mathrm{m}$. (d) Pine wood showing treatment with different ratios before (left) and after 35-h UV treatment (right).

\section{Materials and methods}

Neodol (Neo) was obtained from Shell (Neodol 91-8, M02783) and Xanthan Gum (XG) was obtained from Jungbunzlauer. Araucomex-Pinus radiata and Rhodamine B dye used for wood mimicking and rapid prediction experiments were obtained from Sigma Aldrich (CAS Number 81-88-9). Mica substrates (Highest Grade V1 AFM Mica Discs, $10 \mathrm{~mm}$ ) were purchased from Ted Pella, Inc. Filter Paper with diameter $90 \mathrm{~mm}$ was purchased from Whatman (CAT No. 10312209, Lot No. A10077930). All reagents and chemicals were used without purification and deionized water was used in all experiments.

\section{Biopolymer film preparation on mica}

Xanthan Gum Powder $(0.23 \%$ and $0.45 \%)$ was mixed with Neodol (1.4\% and $2 \%)$ solution (w/v) in the appropriate ratios (4.5-1, 1-1, 1-4.4, 1-6.5 1-11.1, and 1-22.2) and then suspended in deionized water to an equal total volume. If needed, solutions were placed in a $30^{\circ} \mathrm{C}$ oven for $10 \mathrm{~min}$ to aid in complete dissolving of xanthan gum such that no visible aggregates were observed floating in solution. For sample preparation on mica and layer by layer deposition, 1-6.5 XG:Neo was achieved by deposition
$(20 \mu \mathrm{L})$ on the substrate of solution and allowing it to drying. Once dry, the second layer was applied and so on until roughly 5 depositions were achieved for a total of $100 \mu \mathrm{L}$ of solution.

\section{Atomic force microscopy}

For film preparation on mica, a fresh and cleaved piece of mica was mounted on a magnetic disk, allowed to dry and imaged pre-treatment. $25 \mu \mathrm{L}$ of each sample: (1) Xanthan Gum (XG), (2) Neodol (Neo), (3) 4.5:1 (XG:Neodol), (4) $1-6.5$, (5) 1-11.1, and (6) 1-22.2 were applied on the mica and allowed to form a film and dry overnight. For sample preparation on filter paper (cellulose-mimicking material), a piece of filter paper was mounted on a magnetic disk with Epoxy glue, allowed to dry and imaged pre-treatment. 25 $\mu \mathrm{L}$ of each sample: (1) XG, (2) Neo (3), 4.5-1 (XG:Neo), (4) $1-6.5$, (5) 1-11.1, and (6) 1-22.2 were applied on filter paper and allowed to dry overnight. For Atomic Force Quantitative Analysis and Mechanical Mapping, samples were imaged by placing on a magnetic disk and mounting on the J-scanner of the Multimode 8 with a Nanoscope V controller (Bruker). Samples were imaged using PeakForce-Quantitative Nanomechanical Mapping (PeakForce QNM) in air at a scan rate of 


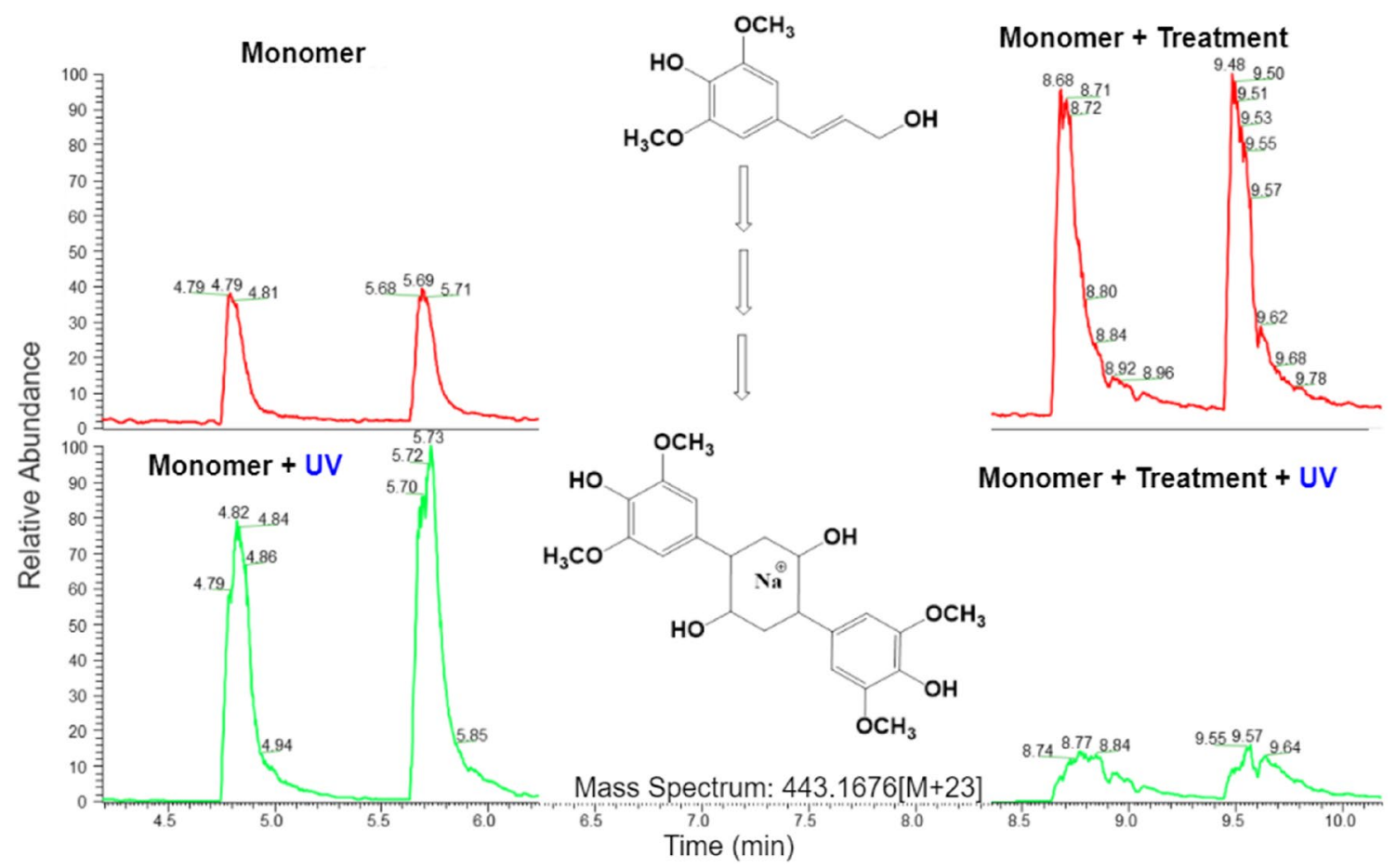

Figure 9: The chromatography of total ions on top and the extracted ion of the degradant compound after UV treatment at $\mathrm{m} / \mathrm{z}=443.1676$ Daltons with a sodium ion adduct $[\mathrm{M}+\mathrm{Na}]+$. All the data were collected in positive detection mode.

0.5-1.5 Hz. The AFM probe utilized in all imaging analysis was a $\mathrm{qp}-\mathrm{HBC}$-SPL Heart Beat Cantilever by Nanosensors. The characteristics of the implored probe include a resonant frequency of $60 \mathrm{kHz}$, a spring constant of $0.5 \mathrm{~N} / \mathrm{m}$, and nominal radius of $<10 \mathrm{~nm}$. For each sample, 3-5 different areas were tested. Representative images are chosen. Images were acquired at $15 \mu \mathrm{m}$ and $5 \mu \mathrm{m}$ scales, aspect ratio of 1.00 , and either 256 or 512 data points/line. Once images were obtained, processing was done using the NanoScope Analysis Software v. 1.5 (Bruker). All plane-fitting and image processing were consistent through all images analyzed (Plane-Fit and 2 nd order flatten). The pore size was quantified using the sectioning analysis within the NanoScope Analysis Software. First, each image had a 1 st order plane fit and a 2 nd order flatten to remove any tilt of the sample. Then, the section analysis tool within the software allows for the display of the cross-sectional profile when a line is drawn on the image. Included in this cross-sectional profile is the radius of the line drawn. $50-75$ pores for each differing ratio is averaged together using this profile.

\section{Rheology}

Rheological properties were characterized in terms of viscoelastic moduli, $G^{\prime}$ (storage or elastic) and $G^{\prime \prime}$ (loss or viscous) measured in oscillatory (dynamic) experiments. The measurements were performed on a RGG2 rheometer by TA
Instruments using $40 \mathrm{~mm} 2$ degrees cone-plate geometry. Two tests were performed for each sample: frequency sweep and temperature sweep. The former was performed at $25^{\circ} \mathrm{C}$ while sweeping angular frequency from 10 to $0.03 \mathrm{rad} / \mathrm{s}$, the latter at $6.28 \mathrm{rad} / \mathrm{s}$ while sweeping temperature from 25 to $60{ }^{\circ} \mathrm{C}$, then back to 25 at $3.5^{\circ} \mathrm{C} / \mathrm{min}$ (hence, $10 \mathrm{~min}$ per sweep). All measurements were performed in the linear regime, i.e., keeping strain amplitude in the range in which moduli are strain-independent (which for these gels was at 3\%).

\section{Electron paramagnetic resonance}

CW EPR measurements were carried out using a X-band spectrometer operating at $9.8 \mathrm{GHz}$ (EMX, Bruker Biospin, Billerica, MA) and a dielectric cavity (EP 4123D, Bruker Biospin, Billerica, MA) at room temperature. Samples were prepared by soaking the pinewood pieces (of equal size) into the XG:Neo mixture for $30 \mathrm{~min}$ and the wood was dried for $1 \mathrm{~h}$ prior to the measurement. The wood sample was then loaded into a quartz tube for measurement. A UVC light source at $254 \mathrm{~nm}$ was mounted outside the resonator, such that the sample could be tested with and without UV irradiation. CW spectra were acquired by using $9 \mathrm{mM}$ of microwave power, 0.5 gauss modulation amplitude, 300 gauss sweep width, and 4 scans for signal averages. The recorded spectra were subjected to determine the relative amount of free radical using peak height. 


\section{Solutions preparation and application on wood}

To understand the final product's performance, a screening design varying all materials with UV protection characteristics was conducted; Neo was varied from 0 to $3 \%$, XG from 0 to $0.92 \%$, and a color solution protecting agent Benzotriazolyl Dodecyl $p$-Cresol was varied from 0 to $0.024 \%$. Results were analyzed with Minitab ${ }^{\star} 18.1$. As for the ratio-dependent evaluation, various XG-Neo solutions were tested (ratios 4.5-1, $1-1,1-4.4,1-6.5,1-11.1,1-22.2$, XG (0.23\% and $0.46 \%)$, Neo $(0.1 \%, 1.5 \%$, and $2.0 \%)$, as well as a deionized water blank and an untreated blank). For solution testing, raw pine wood planks were sectioned into 1.5 in squares, with $500 \mu \mathrm{L}$ of each solution subsequently applied. Upon saturation of the surface, the sample is allowed to dry.

\section{UV chamber exposure}

Samples were exposed to UV light in the Q-SUN Chamber $\left(64^{\circ} \mathrm{C}, 0.35 \mathrm{~W} / \mathrm{m}^{2}, 340 \mathrm{~nm}\right)$ for several hours. $35 \mathrm{~h}$ of exposure time were appropriately divided into two cycles of application, drying, exposure, cleaning, and colorimeter lecture. For the ratiodependent evaluation a total of 40-h exposure time, divided into two cycles of application, exposure, cleaning, and color determination is performed. Specifically, $250 \mu \mathrm{L}$ of the proper solution is applied twice to each square and exposed $(40 \mathrm{~h}, \sim 11$ Mexico sun days) to UV. The wood plank is divided in 4 columns (A, B, $\mathrm{C}$, and $\mathrm{D})$, and 7 rows $(1,2,3,4,5,6$, and 7$)$ whereby solutions are assigned through an Excel randomization. Deionized water blank and untreated blank were used as comparative controls for color change through the weathering of wood.

\section{Colorimeter}

Wood aging was studied through the color change of raw pine wood planks. Several experiments were conducted. Colorimeter measurements were reported and treated in the CIELAB color space. Colorimeter (spectro-guide 45/0 gloss, BYK-Gardner $\mathrm{GmbH}$, Germany) lectures were obtained by calculating the average of each square space $(N=5)$, for all 28 samples. CIELAB color difference was calculated based on the distance between the color locations of the samples. $\mathrm{d} E^{\star}$ was calculated using the initial lecture and the lecture of $n$ cycle.

\section{Liquid chromatography-mass spectrometry (LC-MS)}

Five samples were included: sinapyl alcohol monomer without UVC treatment, sinapyl alcohol after UVC treatment, sinapyl alcohol with XG, sinapyl alcohol with Neo, and sinapyl alcohol with both XG-Neo. The concentrations were made at about 200 ppm and run in duplicate. No further sample preparation is needed before directly injecting the samples to a mass spectrometer. LC analysis was performed using a Thermo Vanquish HPLC System equipped with a binary pump, a thermostated autosampler, an online degasser, and a thermostated column compartment (Thermo Fisher Scientific, San Jose, CA, USA). No column separation was performed; the $\mathrm{LC}$ was used to deliver a mobile phase composed of $50 \% \mathrm{MeOH}-$ Water for MS signal detection. Five samples were injected from a divert valve installed between the LC and ESI probe. MS analysis was performed using a Q-Exactive ${ }^{\text {ru }}$ Hybrid Quadrupole-Orbitrap $^{\text {Ts }}$ mass spectrometer equipped with a HESI-II interface (Thermo Fisher Scientific, San Jose, CA, USA). Samples were analyzed in full scan MS mode from 80 to $1200 \mathrm{~m} / \mathrm{z}$ under positive polarity with heated electrospray ionization. Data were acquired with Xcalibur Software (Version 4.1). The resolution was set at 70,000 FWHM with an automatic gain control (AGC) target at $3 \mathrm{e} 6$ and $200 \mathrm{~ms}$ of maximum ion injection time. The optimized equipment parameters were set as follows: sheath, auxiliary, and curtain gas flow at 35,10 , and 8 , respectively; spray voltage $3.75 \mathrm{kV}$; capillary temperature $320^{\circ} \mathrm{C}$; S-lens RF level 50; auxiliary gas heater temperature $400^{\circ} \mathrm{C}$.

\section{Acknowledgments}

The authors would like to thank Professor Hiroshi Matsui and Dr. Alexei M. Tyryshkin for their guidance, support, and unparalleled subject matter expertise.

\section{Author contributions}

Conceptualization: AS, LP, and KF; methodology, formal analysis and investigation: KF, AS, LP, IR, AP, CC, YW, NL, and $\mathrm{ZH}$; Writing-original draft preparation: KF and AS; Writingreview and editing: KF, AS, and all; All authors have read and agreed to the published version of the manuscript.

\section{Funding}

This research was funded by Colgate-Palmolive Co.

\section{Data availability}

All data generated or analyzed during this study are included in this published article [and its supplementary information files].

\section{Declarations}

Conflict of interest The authors declare that they have no conflict of interest.

\section{Open Access}

This article is licensed under a Creative Commons Attribution 4.0 International License, which permits use, sharing, adaptation, distribution and reproduction in any medium or format, 
as long as you give appropriate credit to the original author(s) and the source, provide a link to the Creative Commons licence, and indicate if changes were made. The images or other third party material in this article are included in the article's Creative Commons licence, unless indicated otherwise in a credit line to the material. If material is not included in the article's Creative Commons licence and your intended use is not permitted by statutory regulation or exceeds the permitted use, you will need to obtain permission directly from the copyright holder. To view a copy of this licence, visit http://creativecommons.org/ licenses/by/4.0/.

\section{Supplementary Information}

The online version contains supplementary material available at https://doi.org/10.1557/s43578-021-00348-6.

\section{References}

1. L. Shuai, M.T. Amiri, Y.M. Questell-Santiago, F. Héroguel, Y. Li, H. Kim, R. Meilan, C. Chapple, J. Ralph, J.S. Luterbacher, Formaldehyde stabilization facilitates lignin monomer production during biomass depolymerization. Science 354(6310), 329-333 (2016). https://doi.org/10.1126/science.aaf7810

2. M. Talebi Amiri, G.R. Dick, Y.M. Questell-Santiago, J.S. Luterbacher, Fractionation of lignocellulosic biomass to produce uncondensed aldehyde-stabilized lignin. Nat. Protoc. 14(3), 921-954 (2019). https://doi.org/10.1038/s41596-018-0121-7

3. B. Tyliszczak, K. Pielichowski, Novel hydrogels containing nanosilver for biomedical applications-synthesis and characterization. J. Polym. Res. 20, 191 (2013)

4. I.M. El-Sherbiny, M.H. Yacoub, Hydrogel scaffolds for tissue engineering: progress and challenges. Glob. Cardiol. Sci. Pract. 3, 316-342 (2013). https://doi.org/10.5339/gcsp.2013.38

5. M.M. Pakulska, B.G. Ballios, M.S. Shoichet, Injectable hydrogels for central nervous system therapy. Biomed. Mater. 7(2), 024101 (2012). https://doi.org/10.1088/1748-6041/7/2/024101

6. M.A. Kaisar, R.K. Sajja, S. Prasad, V.V. Abhyankar, T. Liles, L. Cucullo, New experimental models of the blood-brain barrier for CNS drug discovery. Expert Opin. Drug Discov. 12(1), 89-103 (2017). https://doi.org/10.1080/17460441.2017.125367

7. M. Mir, M.N. Ali, A. Barakullah, A. Gulzar, M. Arshad, S. Fatima, M. Asad, Synthetic polymeric biomaterials for wound healing: a review. Prog. Biomater. 7(1), 1-21 (2018). https://doi. org/10.1007/s40204-018-0083-4

8. X. Zhang, Y. Guan, Y. Zhang, Ultrathin hydrogel films for rapid optical biosensing. Biomacromolecules 13(1), 92-97 (2012). https://doi.org/10.1021/bm2012696

9. R. Niladri, S. Nabanita, S. Petr, Biodegradable hydrogel film for food packaging, in Proceedings of the 4th WSEAS International Conference on Energy and
Development-Environment-Biomedicine (WSEAS Press, Athens, Greece, 2011), pp. 329-334

10. E. Sason, M. Kolitz-Domb, J.H. Chill, S. Margel, Engineering of durable antifog thin coatings on plastic films by UV-curing of proteinoid prepolymers with PEG-diacrylate monomers. ACS Omega 4, 9352-9360 (2019). https://doi.org/10.1021/acsomega. $9 \mathrm{~b} 00336$

11. X. Zhang, J. He, Hydrogen-bonding-supported self-healing antifogging thin films. Sci. Rep. 5, 9227 (2015). https://doi.org/ 10.1038/srep09227

12. H. Chen, F. Yang, Q. Chan, J. Zheng, A novel design of multimechanoresponsive and mechanically strong hydrogels. Adv. Mater. 29, 1606900 (2017). https://doi.org/10.1002/adma.20160 6900

13. B. Chollet, M. Li, E. Martwong, B. Bresson, C. Fretigny, P. Tabeling, Y. Tran, Multiscale surface-attached hydrogel thin films with tailored architecture. ACS Appl. Mater. Interfaces 8(18), 11729-11738 (2016). https://doi.org/10.1021/acsami.6b00446

14. X. Zhaoyang, L. Jianyu, Z. Huan, J. Xiangdong, Y. Chuang, W. Fei, P. Yuanyuan, L. Nana, L. Xiaoyan, S. Linja, S. Xiaomei, Morphological and swelling behavior of cellulose nanofiber (CNF)/ poly(vinyl alcohol) (PVA) hydrogels: poly(ethylene glycol) (PEG) as porogen. RSC Adv. 6, 43626-43633 (2016). https://doi.org/10. 1039/C6RA03620A

15. M.H. Sheridan, L.D. Shea, M.C. Peters, D.J. Mooney, Bioabsorbable polymer scaffolds for tissue engineering capable of sustained growth factor delivery. J. Control. Release Off. J. Control. Release Soc. 64(1-3), 91-102 (2000). https://doi.org/10.1016/s01683659(99)00138-8

16. R.A. Quirk, R.M. France, K.M. Shakesheff, S.M. Howdle, Supercritical fluid technologies and tissue engineering scaffolds. Curr. Opin. Solid State Mater. Sci. 8, 313 (2004). https://doi.org/10. 1016/j.cossms.2003.12.004

17. N. Annabi, J.W. Nichol, X. Zhong, C. Ji, S. Koshy, A. Khademhosseini, F. Dehghani, Controlling the porosity and microarchitecture of hydrogels for tissue engineering. Tissue Eng. Part B Rev. 16(4), 371-383 (2010). https://doi.org/10.1089/ten.TEB.2009. 0639

18. L. Li, Y.L. Hsieh, Ultra-fine polyelectrolyte hydrogel fibres from poly(acrylic acid)/poly(vinyl alcohol). Nanotechnology 16, 2852 (2005). https://iopscience.iop.org/article/. https://doi.org/10. 1088/0957-4484/16/12/020

19. F. García-Ochoa, V.E. Santos, J.A. Casas, E. Gómez, Xanthan gum: production, recovery, and properties. Biotechnol. Adv. 18(7), 549-579 (2000). https://doi.org/10.1016/s0734-9750(00) 00050-1

20. V.S. Kulkarni, C. Shaw (eds.), Essential Chemistry for Formulators of Semisolid and Liquid Dosages (Academic Press, Boston, 2016), pp. 43-69. https://doi.org/10.1016/B978-0-12-801024-2.00005-4

21. S.M.M. Gheller, C.D. Corcini, F.C.C. Santos, G.C. Tavares, V.G.G. Costa, B.R. Curcio, C.E.W. Nogueira, A.S. Varela Junior, Arq. 
Bras. Med. Vet. Zootec. 71(1), 28-34 (2019). https://doi.org/10. 1590/1678-4162-10117

22. G.D. Gastal, E.F. Silva, B. Mion, A.S. Varela Junior, C.E. Rosa, C.D. Corcini, R.G. Mondadori, A.D. Vieira, I. Bianchi, T. Lucia Jr., Antioxidant effect of xanthan gum on ram sperm after freezing and thawing. Cryo Letters 38(3), 187-193 (2017). https:// pubmed.ncbi.nlm.nih.gov/28767741/

23. C. Amico, T. Tornetta, C. Scifo, A.R. Blanco, Antioxidant effect of $0.2 \%$ xanthan gum in ocular surface corneal epithelial cells. Curr. Eye Res. 40(1), $72-76$ (2015). https://doi.org/10.3109/02713683. 2014.914542

24. S.A. Rather, F.A. Masoodi, R. Akhter, J.A. Rather, A. Gani, S.M. Wani, A.H. Malik, Application of guar-xanthan gum mixture as a partial fat replacer in meat emulsions. J. Food Sci. Technol. 53(6), 2876-2886 (2016). https://doi.org/10.1007/s13197-016-2270-4

25. V. Krstonosic, M. Milanovic, L. Dokic, Application of different techniques in the determination of xanthan gum-SDS and xanthan gum-Tween 80 interaction. Food Hydrocoll. 87, 108-118 (2019). https://doi.org/10.1016/j.foodhyd.2018.07.040

26. J. Maitra, V. Kumar Shukla, Cross-linking in hydrogels: a review. Am. J. Polym. Sci. 4(2), 25-31 (2014). https://doi.org/10.5923/j. ajps.20140402.01

27. M. Zhang, Y. Li, P.V. Kolluru, C. Brinso, Determination of mechanical properties of polymer interphase using combined atomic force microscope (AFM) experiments and finite element simulations. Macromolecules 51(20), 8229-8240 (2018)

28. R.T. De Silva, P. Pasbakhsh, K.L. Goh, S.-P. Chai, J. Chen, Synthesis and characterization of poly (lactic acid)/halloysite bionanocomposite films. J. Compos. Mater. 48(30), 3705-3717 (2014)

29. P. Paquin (ed.), Functional and Speciality Beverage Technology 2009 (Woodhead Publishing, 2009)
30. S.N. Goyanes, N.B. D’Accorso, in Industrial Applications of Renewable Biomass Products Past, Present and Future, ed. by S.N. Goyanes, N.B. D’Accorso (Springer International Publishing, 2017), pp. 210-213

31. Y. Liu, W. He, Z. Zhang, B. Lee, Recent developments in tough hydrogels for biomedical applications. Gels 4(2), 46 (2018). https://doi.org/10.3390/gels4020046

32. M. Lerner, Replacing Carpet With Hardwood Floor: Which Has a Better Resale Value? (2014)

33. W.C. Feist, D.N.S. Hon, in The Chemistry of Solid Wood, ed. by R. Rowell (American Chemical Society, 1984), pp. 401-451

34. R. Flatt, I. Schober, in Understanding the Rheology of Concrete, ed. by N. Roussel (Woodhead Publishing, 2012), pp. 144-208

35. W.C. Feist, in Archaeological Wood. Outdoor Wood Weathering and Protection (American Chemical Society, 1989), pp. 263-298. https://pubs.acs.org/doi/abs/. https://doi.org/10.1021/ba-19900225.ch011

36. S.H. Ghaffar, M. Fan, Lignin in straw and its applications as an adhesive. Int. J. Adhes. Adhes. 48, 92-101 (2014). https://doi.org/ 10.1016/j.jadhadh.2013.09.001

37. Y. Zhang, D. Legland, F. El Hage, M.F. Devaux, F. Guillon, M. Reymond, V. Méchin, Changes in cell walls lignification, feruloylation and p-coumaroylation throughout maize internode development. PLoS One 14(7), e0219923 (2019). https://doi.org/ 10.1371/journal.pone.0219923

38. R.A. Malvar, A. Burtón, B. Ordás, R. Santiago, in Crop Protection in Research Advances, ed. by E.N. Burton, P.V. Williams (Nova Science Publishers, Inc., New York), p. 67

39. R.B. Santos, E.A. Capanema, M.Y. Balakshin, H.M. Chang, H. Jameel, Lignin structural variation in hardwood species. J. Agric. Food Chem. 60(19), 4923 (2012) 